\title{
Gesundheitsförderung in der Grundausbildung von Lehrerinnen und Lehrern in der Schweiz
}

\begin{abstract}
Zusammenfassung
Die Schule ist in verschiedener Hinsicht ein für Gesundheitsforderung geradezu orädestinierter Ort. Deshalb kommt auch der Vorbereitung zukünftiger Lehrkräfte auf ihre Aufgaben im Bereich der Gesundheitsförderung eine grosse Bedeutung zu. Trotz positiver Einzelbeispiele und einer Anzahl von engagierten VorkämpferInnen für die Verankerung von Gesundheitsförderung in der Grundausbildung von Lehrkräften weisen die Ergebnisse einer Umfrage bel den schweizerischen Lehrerbildungsinstituten darauf hin, dass in rund $30 \%$ der Ausbildungsstätten Gesundheitsthemen keinen expliziten Platz haben Auch in den anderen Instituten ist die Stellung der Beauftragten flu Gesundheitsförderung oft marginal. Die durch die Untersuchung intendierten Auswirkungen in der Praxis wurden nur teilweise realisiert: Zwar wurden die Ergebnisse von der Schweizerischen Konferenz der kantonalen Erziehungsdirektoren publiziert doch ist es nicht gelungen, eine weiterführende Gruppe von Beauftragten für Gesundheitsförderung an den schweizerischen Lehrerbildungsinstituten zu konstituieren.
\end{abstract}

Die Schule ist ein geeigneter Ort zur Verbesserung der Gesundheit von Schülerinnen und Schülern, ihrer Familien, der Angestellten der Schule und der Bevölkerung der Gemeinde. Die Schule kann grundlegende Anliegen im Bereich der Erziehung und der Gesundheit unterstützen. Im "Setting" Schule bieten sich Möglichkeiten, auch mit knappen Ressourcen zu signifikanten Verbesserungen in den Bereichen Gesundheit und Erziehung zu gelangen ${ }^{1}$. Für die Diskus- sion und Abgrenzung der Begriffe Gesundheitserziehung, Gesundheitsförderung und Prävention sei auf den diesem Artikel zugrundeliegenden Bericht verwiesen ${ }^{2}$. Die folgenden drei Handlungsperspektiven für die Gesundheitsförderung in Schulen lassen sich aus den Zielsetzungen der Charta von Ottawa ablesen ${ }^{3}$ :

Anwaltschaftliche Vertretung von gesundheitsbezogenen Interessen von Schülerinnen und Schülern, welche von Schulleitungen und Lehrkräften den möglichst weitgehenden Einsatz für das körperliche, psychische und soziale Wohlbefinden fordert. Die Organisation des Schulunterrichts könnte vermehrt nach solchen Kriterien ausgerichtet werden; von der Schulleitung wird aber auch eine engagierte und fachkundige Stellungnahme erwartet, wenn es um raumund ortsplanerische Entscheidungen wie Sport- und Freizeitanlagen, Velowege oder Jugendtreffs geht.

Befähigung von Jugendlichen zur Verwirklichung ihres grösstmöglichen Gesundheitspotentials; dies umfasst - gemäss dem Konzept der Gesundheitsförderung - auch die gemeinsame, praxisbezogene Erarbeitung von Problemeinsichten und von Handlungskompetenzen.

Herstellung und Erleichterung von Kontakten sowie Vernetzung mit anderen auf Gemeindeebene tätigen Organisationen und Institutionen; die Arbeit von Jugendämtern, Beratungsstellen, Sportvereinen etc. könnte im Unterricht diskutiert werden; gemeinsame Veranstaltungen könnten übergreifende Gesundheitsthemen in der Öffentlichkeit zur Diskussion stellen. Lehrerinnen und Lehrern kommt in der Schule eine zentrale Rolle 
zu; dies gilt selbstverständlich auch für den Bereich der Gesundheitsförderung in Schulen. Lehrkräfte sind die "Manager" der Organisation Schule, und so liegt auch bei der Gesundheitsförderung eine grosse Verantwortung in ihrer Hand. Niemand sonst hat wie sie einen Gesamtüberblick über das Schulgeschehen. Als Erwachsenen kommt ihnen auch eine Rolle als Vorbild für die Kinder und Jugendlichen zu. Im Bereich der Gesundheitsförderung sind die Lehrkräfte die Hauptverantwortlichen für die Gesundheitserziehung; im Bereich der Prävention kommt ihnen eine wichtige Rolle in der Vermittlung zwischen ihren Schülerinnen und Schülern und Präventionsfachleuten zu. Zudem übernehmen sie eine Art "Triage Funktion", indem sie bei besonderen Gesundheitsproblemen (mit anderen) für die Weitervermittlung der Betroffenen an eine spezialisierte Stelle zuständig sind. Wie jede Verantwortung trägt auch diese zu einem positiven Selbstverständnis und einer erhöhten Arbeitszufriedenheit bei. Sie birgt aber auch problematische Seiten: Neue Verantwortungsbereiche werden der Schule - und damit in erster Linie den Lehrpersonen übertragen, ohne dass es in der Regel in einem anderen $\mathrm{zu}$ einem Abbau oder einer Entlastung kommt. Diese additiven Verfahrensweisen können $\mathrm{zu}$ zeitlicher und inhaltlicher Überlastung führen $^{4}$.

In der Praxis begegnet man oft engagierten Lehrerinnen und Lehrern, die sich voller Überzeugung und Enthusiasmus in das Abenteuer "Gesundheit" stürzen, um später ausgepumpt und frustriert nicht selten auch unter ernsten Gesundheitsproblemen leidend aufzugeben. Noch zu häufig handeln Lehrkräfte als Einzelkämpferinnen und -kämpfer. Eine einzelne Lehrperson kann wohl eine Initiative anregen, aber zur Umsetzung von Gesundheitsförderung in Schulen braucht es das Kollegium ${ }^{5}$.
Gesundheitsförderung in Schulen hat für Lehrerinnen und Lehrer aber noch eine weitere Dimension: Es geht auch um ihre eigene Gesundheit an ihrem Arbeitsplatz, der Schule. Lehrerinnen und Lehrer sind also zentrale Akteure von Gesundheitsförderung in Schulen in dreierlei Hinsicht:

- In Bezug auf ihre pädagogische und multiplikatorische Funktion in der Gesundheitserziehung und bezüglich ihrer Vermittlungsfunktion in der Prävention.

- Im Hinblick auf die Entwicklung der Organisation Schule.

- Im Hinblick auf die Förderung ihrer eigenen Gesundheit am Arbeitsplatz Schule und untereinander im Kollegium.

Die Grundausbildung, meist in einem Zug genannt mit Fort- und Weiterbildung, taucht als notwendige Voraussetzung zur Realisierung von Gesundheitsförderung in Schulen und als auszubauende Perspektive bei Pilotprojekten regelmässig auf. Deshalb erschien es sinnvoll, in einem ersten Schritt die Situation der Gesundheitsförderung in der Grundausbildung schweizerischer Lehrkräfte in Form einer Bestandesaufnahme zu erfassen. Die Sensibilisierung der betroffenen Organe (Schweizerische Konferenz der kantonalen Erziehungsdirektoren (EDK), Lehrerbildungsinstitute in den Kantonen) für das Anliegen der Gesundheitsförderung in Schulen war ein weiteres Ziel der Untersuchung. Als modellhafte Beispiele wurden ausserdem das Pilotprojekt „Gesundheitserziehung und Suchtprävention am Seminar Thun" (Kanton Bern), der Ausbildungsgang „Education générale et sociale (EGS)“ am Institut pédagogique Porrentruy (Kanton Jura) und der Kurs "Adolescence: stress, déviances et facteurs protecteurs" (Basis- und Vertiefungskurs) am Séminaire pédagogique de l'enseignement secondaire (SPES) (Kanton Waadt) bezüglich Inhalt, Form, Rahmenbedingungen und Evaluation beschrieben. Die Auswahl dieser drei Modelle erfolgte aufgrund ihres innovativen Charakters und ihres umfasssenden Ansatzes sowie als Beispiele unterschiedlicher Lehrerbildungssysteme aus verschiedenen Sprachregionen ${ }^{2}$.

\section{Methode}

Das Ziel der Umfrage bei den schweizerischen Lehrerbildungsinstituten war es, einen Überblick ïber aktuelle und geplante Aktivitäten in den Kantonen geben zu können. Die so entstandene Übersicht ist einerseits ein Instrument zur Analyse der Situation; andererseits dient sie als Vergleichs- und Informationsbasis, da Lehrbeauftragte für Gesundheitsförderung in den Lehrerbildungsinstituten bisher kein institutionalisiertes Austauschforum auf schweizerischer oder regionaler Ebene kennen.

Für die Erstbefragung wurden die bei der EDK vorhandenen Adressen der schweizerischen Lehrerbildungsinstitutionen benutzt. Dieser Adressen-Satz umfasste 130 Adressen, 102 (78\%) aus der deutschen Schweiz, 28 (22\%) aus der französischen Schweiz und dem Tessin. Im Begleitbrief wurden die Schulleitungen gebeten, den Fragebogen an die für den Bereich Gesundheit zuständige Person in ihrer Schule weiterzuleiten. Neben Fragen zum Einbezug der Gesundheitsförderung an den Lehrerbildungsinstituten enthielt der Fragebogen auch eine Abklärung des Interesses an einer Austauschtagung unter den Lehrbeauftragten für Gesundheitsförderung und -erziehung in den Lehrerbildungsinstituten. Es war geplant, bei genügendem Interesse eine bis zwei solcher Tagungen durchzuführen. Aufgrund der Reaktionen auf den Fragebogen wurden dann in der deutschen Schweiz zwei Austauschtagungen (im Mai und im 
September 1995) durchgeführt. An der Austauschtagung vom September 1995 äusserten die Teilnehmenden das Bedürfnis, sich als Arbeitsgruppe zu konstituieren und jährlich ein Austausch- und Fortbildungstreffen durchzuführen; ausserdem wurde ein Antrag an die EDK betreffend Gesundheitsförderung in der Lehrerinnen- und Lehrerbildung ausgearbeitet.

In der französischen Schweiz stiess die erste Austauschtagung im Mai 1995 auf ein zahlenmässig so geringes Interesse, dass anschliessend Einzelgespräche statt Gruppentreffen durchgeführt wurden. Ausserdem stellte ein Empfänger des Fragebogens zehn weitere Adressen zur Verfügung, so dass in der französischen Schweiz eine Zweitbefragung per Fragebogen durchgeführt werden konnte. Die Tabelle 1 fasst das realisierte Vorgehen schematisch zusammen.

\section{Resultate}

Rücklauf

Die Erstbefragung ergab mit 53 von 130 zurückgesandten Fragebogen einen Rücklauf von knapp $41 \%$.
11 Fragebogen (7 aus der deutschen Schweiz, 4 aus der romanischen Schweiz) waren nicht auswertbar, namentlich weil es sich bei den Angeschriebenen nicht um eigentliche Lehrerbildungsinstitute handelte. Damit gingen aus der Erstbefragung 42 auswertbare Fragebogen hervor. In der deutschen Schweiz lag der Rücklauf mit 44 $(43 \%)$ zurückgesandten Bogen eindeutig höher als in der romanischen Schweiz mit 9 zurückgesandten Bogen $(32 \%)$. In der Zweitbefragung in der romanischen Schweiz kamen 6 von 10 Fragebogen zurück. So betrug in der Gesamtbefragung die Antwortrate $42 \%$ (59/140 Fragebogen); in der deutschen Schweiz antworteten $43 \%$ (44/102) der angeschriebenen Lehrerbildungsinstitute, in der romanischen Schweiz 39\% (15/38). Zum Schluss lagen 48 auswertbare Fragebogen vor.

Art der antwortenden Lehrerbildungsinstitute

Rund vierzig Prozent der Antworten kamen aus Lehrerbildungsinstituten, an welchen Lehrkräfte für die Primarschule ausgebildet werden, ein knappes Drittel von
Kindergarten-Seminaren und ein gutes Fünftel von Ausbildungsstätten für die Sekundarstufe; den Rest machen Hauswirtschafts- und Seminare für Textiles Arbeiten aus. Interessant sind im Hinblick auf die Aussagekraft der vorliegenden Ergebnisse die Unterschiede bei den Antwortraten: Sie lagen bei den Kindergartenseminaren bei $67 \%$, bei den Ausbildungsstätten für die Primarstufe bei $43 \%$, bei jenen der Sekundarstufe bei $37 \%$ und bei den Hauswirtschaftsund Seminaren für Textiles Arbeiten bei $18 \%$.

\section{Beruflicher Hintergrund der Lehrbeauftragten für Gesundheitsförderung}

Bei den antwortenden Lehrerbildungsinstituten sind zu rund $60 \%$ Personen mit einem pädagogischen Hintergrund für die Gesundheitsförderung verantwortlich. Ein gutes Viertel hat eine medizinisch ausgerichtete Vorbildung; die übrigen Fragebogen insbesondere von Instituten, welche Gesundheitsförderung nicht einbeziehen, sind unter diesem Blickwinkel nicht auswertbar.
Schriftliche Befragung der Lehrerbildungsinstitute (gesamtschweizerisch) (März 1995) deutsche Schweiz:

1. Austauschtagung (Mai 1995)
2. Austauschtagung

(September 1995)

- Befragung von Schlüsselpersonen

- Konstitution einer weiterführenden Gruppe
Telefonische Befragung einer Stichprobe von "Non-Responders" (Januar 1996)

französische/italienische Schweiz:

- Einzelgespräche (Mai 1995)

$$
N=130
$$

- Befragung von Schlüsselpersonen

- schriftliche Zweitbefragung
$\mathrm{N}=\mathbf{2 5}$

Tabelle 1. Vorgehen bei der Befragung der schweizerischen Lehrerbildungsinstitute und Folgeaktionen. 


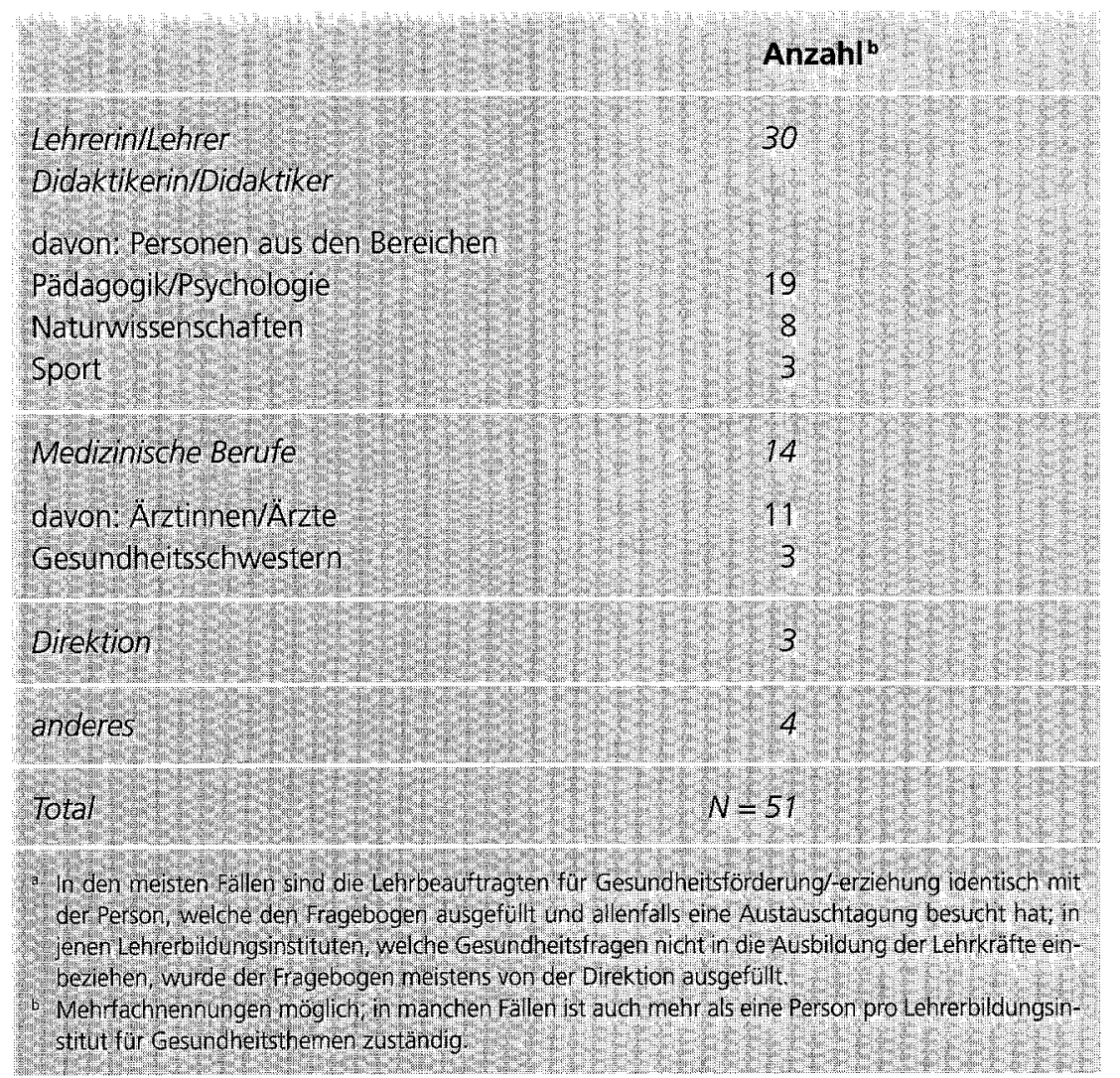

Tabelle 2. Beruflicher Hintergrund der Lehrbeauftragten für Gesundheitsförderung und -erziehung ${ }^{a}$.

Obligatorischer/fakultativer Charakter des Lehrangebotes in Gesundheit

Zwei Drittel der 48 Antwortenden kennen in ihrem Institut ausschliesslich Pflichtveranstaltungen zur Gesundheitsförderung; in zwei Lehrerbildungsinstituten werden Pflicht- und Wahlveranstaltungen verbunden, fünf thematisieren Gesundheit lediglich als Option. Immerhin ein knappes Fünftel der Antwortenden gibt an, Gesundheitsförderung überhaupt nicht einzubeziehen, und dies aus finanziellen resp. damit verbundenen zeitlichen Beweggründen (Prioritätensetzung). Um die Vermutung zu prüfen, die vorliegenden Ergebnisse seien durch positive Selektion verzerrt, wurde bei einer Stichprobe von 25 Non-Responders eine telefonische Nachbefragung der nachfolgende Non-Responder gewählt. Obwohl manche der Non-Responders ein ausführliches Lehrangebot $\mathrm{zu}$ Gesundheit vorweisen, stärkt die Nachbefragung die Annahme, dass die antwortenden Lehrerbildungsinstitute tendenziell über ein überdurchschnittliches Lehrangebot im Bereich Gesundheit verfügen: Gut die Hälfte (gegenüber $80 \%$ bei den Antwortenden) der Non-Responder-Stichprobe kennt den - expliziten oder impliziten - Einbezug von Gesundheit in ihre Ausbildung. Der Anteil der Lehrerbildungsinstitute, welche keinen Einbezug von Gesundheitsthemen kennen, erhöht sich dagegen von knapp $20 \%$ auf fast einen Drittel.*

Form des Einbezugs von Gesundheitsförderung

Bei der Form des Einbezugs von Gesundheitsförderung schliesslich präsentiert sich ein ziemlich aus-

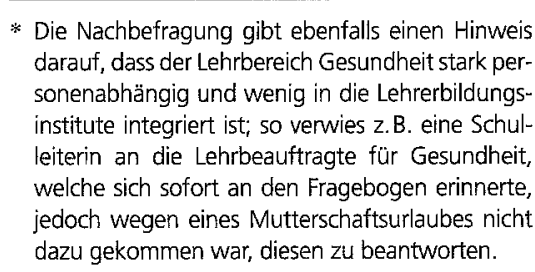
auf einen Responder fiel, wurde

durchgeführt. Dazu wurde auf der Adressliste jede fünfte Anschrift bezeichnet; falls die Bezeichnung

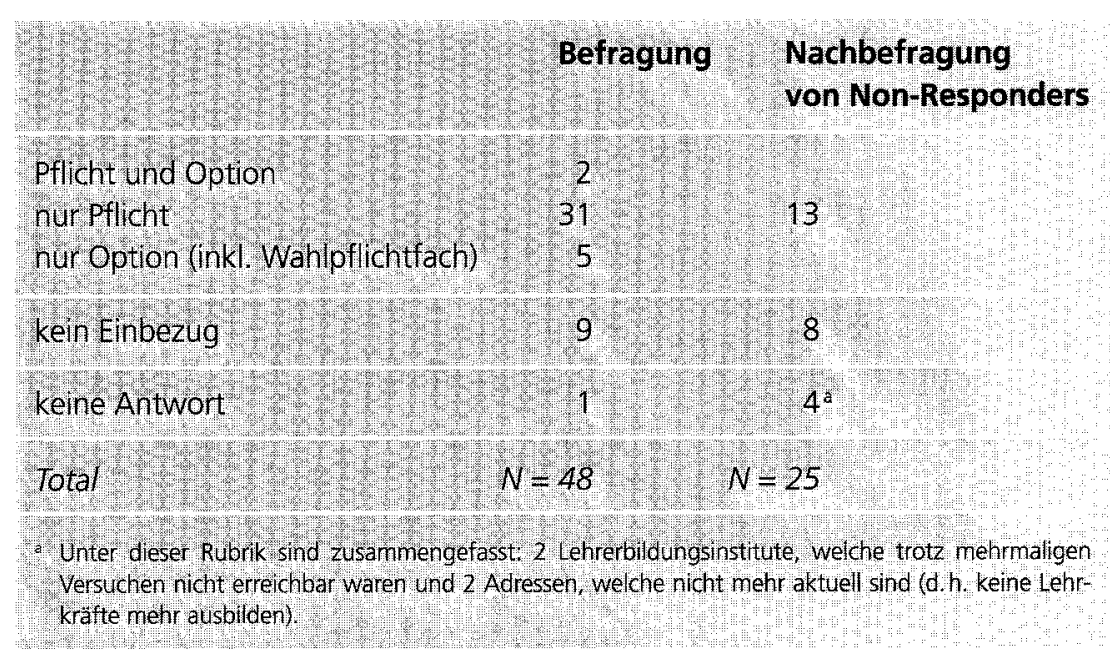

Tabelle 3. Einbezug von Gesundheit im Lehrangebot der Lehrerbildungsinstitute. 
geglichenes Bild: Manche Lehrerbildungsinstitute thematisieren $\mathrm{Ge}$ sundheitsthemen sowohl in Blockveranstaltungen als auch in Wochenstunden $(33 \%)$, andere führen nur Blockveranstaltungen (22\%) oder nur Wochenstunden durch, welche sich implizit oder explizit mit Themen der Gesundheitsförderung befassen ( $45 \%)$.

Behandelte Themen

Das folgende Diagramm gibt eine Übersicht über die in der Grund-

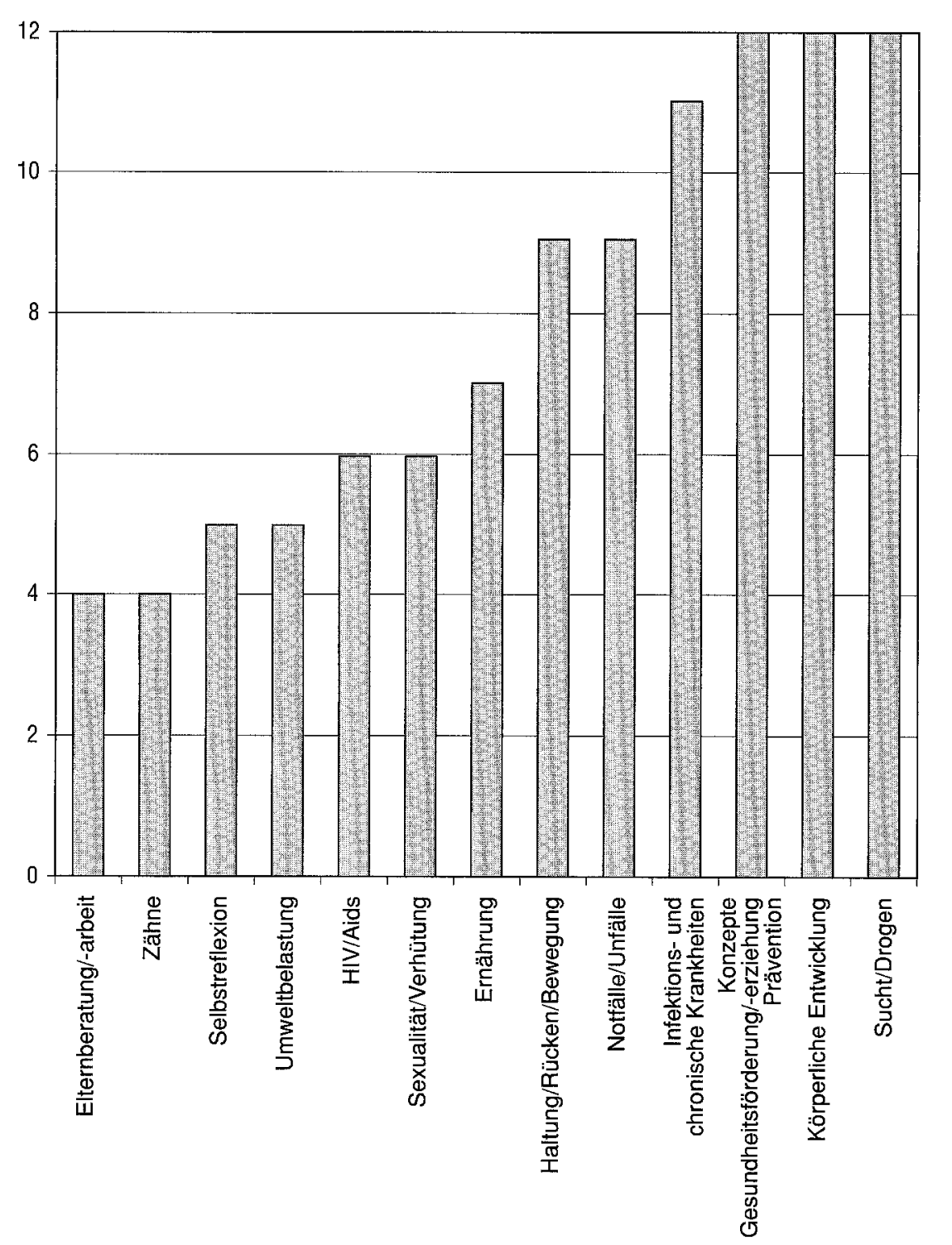

Tabelle 4. Behandelte Themen in der Grundausbildung von Lehrerinnen und Lehrern.

ausbildung von Lehrerinnen und Lehrern behandelten Themen.

\section{Diskussion}

Um den Anforderungen einer gesundheitsfördernden Schule gerecht zu werden, sind Lehrerinnen und Lehrer darauf angewiesen, während ihrer Grundausbildung auch auf jene Aufgaben vorbereitet $\mathrm{zu}$ werden, welche für die Gesundheitsförderung relevant sind. Obwohl die vorliegenden Er- gebnisse weder repräsentativ noch umfassend sind, erlauben sie doch einen Einblick in die aktuelle Situation der Gesundheitsförderung an den schweizerischen Lehrerbildungsinstituten, welche im folgenden diskutiert werden soll.

\section{Positive Selektion in der Befragung}

Mit grösster Wahrscheinlichkeit stellen die vorliegenden Ergebnisse eine positive Selektion dar, da sie nur aufgrund eines freiwilligen Engagements der Befragten erhoben werden konnten. Diese Annahme wird durch die Ergebnisse einer telefonischen Nachbefragung bei einer Stichprobe von 25 Non-Responders gestützt; der Anteil von Lehrerbildungsinstituten, welche Gesundheitsförderung nicht einbeziehen, muss wohl gegen $30 \%$ hin korrigiert werden.

Lehrerbildungsinstitute als gesundheitsfördernde Lernorte

Die Lehrerbildungsinstitute selber sind oftmals keine gesundheitsfördernden Lernorte. Dabei stellen Schulen - und damit auch die Lehrerbildungsinstitute selber wie dargelegt gute Orte zur Erlangung von Kompetenzen dar, die dazu beitragen, das Wohlbefinden $\mathrm{zu}$ verbessern. Gesundheitsförderung kann in verschiedenen Fachbereichen thematisiert werden.

Gesundheitsförderung erfordert eine doppelte Fachkompetenz und Interdisziplinarität

Gesundheitsförderung in Schulen verlangt eine doppelte Fachkompetenz: einerseits im inhaltlichen Bereich der Gesundheitsförderung, andererseits bezüglich der Vermittlung dieser Inhalte. Lehrkräfte, welche dazu in ihrer Grundausbildung befähigt werden, sind auch Fachleute der Gesundheitsförderung. Dabei gibt es jedoch im Bereich der Gesundheitsförderung keine Fachdidaktik, wie man sie in 
anderen Fächern (z. B. Muttersprache, Fremdsprachen, Mathematik) kennt.

Laut den vorliegenden Ergebnissen liegt die Verantwortung für die Gesundheitsförderung in den Lehrerbildungsinstituten in den meisten Fällen (rund 60\%) bei Personen mit pädagogischem Hintergrund und nur in einem guten Viertel $(27 \%)$ bei medizinisch vorgebildetem Personal. Vor diesem Hintergrund ist festzuhalten, dass die Vertreterinnen und Vertreter medizinischer Berufe an den beiden Austauschtagungen in der deutschen Schweiz ,überrepräsentiert" waren. Dies ist umso erstaunlicher, als gerade diese Personen meist in einem losen Verhältnis $\mathrm{zu}$ ihrem Institut stehen und sich meistens nur für den gesundheitsspezifischen Unterricht dort aufhalten. Die Austauschtagungen besuchten sie grösstenteils während ihrer Freizeit, respektive ohne finanzielle Abgeltung durch ihr Lehrerbildungsinstitut. Ist ihre beträchtliche Präsenz an den Austauschtagungen ein reiner Zufall? Sind sie in dieser Frage mehr sensibilisiert oder ist ihr Leidensdruck grösser? An dieser Stelle lassen sich dazu nur Vermutungen anstellen.

Obwohl gerade der Bereich der Gesundheitsförderung viel Raum lässt für unterschiedliche und innovative Unterrichtsformen, werden die Chancen zur gelebten Interdisziplinarität noch relativ wenig genutzt; dies liegt teilweise daran, dass keine geeigneten Zeitgefässe für solche Projekte zur Verfügung stehen. Somit ist die Koordination der Gesundheitsförderung mit den übrigen Aktivitäten in den Lehrerbildungsinstituten oft nicht vorhanden, auch bedingt durch die mangelhafte Integration der Lehrbeauftragten für Gesundheitsförderung im Kollegium. Zwar gibt es dank guter persönlicher Kontakte oft Absprachen, welche das Problem lösen. Es fehlt jedoch die strukturelle Absicherung dieser Koordination.
Einzelinitiativen ersetzen nicht eine Struktur

Es gibt viele positive Einzelbeispiele und vielversprechende kantonale Projekte. Das Problem dieser Initiativen liegt jedoch in der Tatsache, dass sie kaum bekannt sind. Der Austausch von positiven und negativen Erfahrungen in Projekten der Gesundheitsförderung in Lehrerbildungsinstituten kommt oftmals zu kurz, weil im föderalistischen politischen System der Schweiz die Kantone für das Erziehungs- und Gesundheitswesen zuständig sind. Diese Situation wird akzentuiert durch die Tatsache, dass die Lehrbeauftragten für Gesundheitsförderung oftmals Personen sind, welche nicht eigentlich in die Lehrerbildungsinstitute integriert sind.

\section{Reflexion ist gefragt}

Eine unabdingbare Voraussetzung für die erfolgreiche Thematisierung von Gesundheitsförderung in Schulen ist die Reflexion der relevanten Fragen und Konzepte durch die Vermittlerinnen und Vermittler, also die Lehrkräfte. Dazu gehört auch die Auseinandersetzung mit der Entwicklung der „traditionellen Gesundheitserziehung" zum umfassenden Konzept der Gesundheitsförderung.

\section{Grundlagen in Lehrplänen} vorhanden - Umsetzung schwierig

In den meisten Fällen finden sich in den Lehrplänen gute Grundlagen für die Gesundheitsförderung in Schulen. Das Problem liegt teilweise bei der mangelnden Kenntnis der geltenden Lehrpläne und vor allem bei der Umsetzung dieser offenen, nicht ganz einfach fassbaren Konzepte; Lösungsansätze zu diesem Problem kommen in der Grundausbildung von Lehrerinnen und Lehrern noch oft zu kurz. Ausserdem entsprechen die während der Ausbildung behandelten
Themen nicht unbedingt jenen, welche später im Unterricht thematisiert werden: In einer Studie wurden 878 Lehrkräfte nach den Themen befragt, welche sie innerhalb der Gesundheitserziehung in ihrem Unterricht behandeln ${ }^{6}$. Dabei fällt auf, dass der Themenbereich Sucht und Drogen viel weiter unten rangiert als in der vorliegenden Umfrage bei den schweizerischen Lehrerbildungsinstituten. "Spitzenreiter" im Schulunterricht scheinen dagegen die Prävention von Unfällen und Zahnhygiene zu sein.*

Der Ausschuss Lehrerbildung der EDK hat in Anlehnung an die, diesem Artikel zugrundeliegende Untersuchung eine Reihe von Massnahmen ins Auge gefasst (Tabelle 5), welche zeigen, dass die mit dieser Arbeit intendierte Sensibilisierung für die Anliegen der Gesundheitsförderung in Schulen allgemein und für die Bedeutung von Gesundheitsförderung in der Grundausbildung von Lehrkräften im Speziellen auf dieser Ebene gelungen ist. Erläuterungen zu diesem Prozess finden sich in ${ }^{2}$.

Auf der Ebene der Lehrbeauftragten für Gesundheitsförderung an den schweizerischen Lehrerbildungsinstituten war diese Umsetzung in der Praxis weniger erfolgreich: Zwar wurde von ihrer Seite wiederholt der Wunsch geäussert, über ein kontinuierliches Austauschforum verfügen zu können, doch zeigte sich schon gegen Ende

\footnotetext{
* Bei Themen wie „körperliche Entwicklung" oder "Infektions- und chronische Krankheiten" leuchtet es selbstverständlich ein, dass sie in der Grundausbildung der Lehrkräfte eine grössere Rolle spielen als später in der Berufspraxis. Ein weiterer Grund für diese Situation könnte sein, dass beispielsweise Fragen der Suchtprävention, der Schwangerschaftsverhütung oder der Aidsprävention vor allem für die angehenden Lehrerinnen und Lehrer selber als wichtig erachtet werden, im Hinblick auf ihre Unterrichtstätigkeit jedoch eine weniger grosse Rolle spielen. Aufgrund der vorliegenden Daten ist es jedoch nicht möglich, dazu genauere Aussagen zu machen.
} 
1. Gesundheitsförderung muss zu einem Anliegen der Lehrerbildung als ganzer werden, denn Gesundheitsförderung ist nicht nur eine Aufgabe einzelner Fächer oder Disziplinen, sondern Ausdruck einer von allen Lehrkräften, $d . h$. auch von allen Dozentinnen und Dozenten einer Lehrerbildungsanstalt getragenen Haltung, verbunden mit den nötigen Kompetenzen, konstruktiv auf gesundheitsfördernde Weise ins Schulgeschehen eingreifen zu können.

2. Lehrerbildungsinstitute sollten selbst als gesundheitsfördernde Lernorte konzipiert werden und Gesundheitsförderung exemplarisch praktizieren.

3. Gesundheitsförderung gehört in jedes Fach, aber damit dieser Gesichtspunkt überhaupt Eingang finden kann, müssen in den Lehrerbildungsinstitutionen spezielle Beauftragte für Gesundheitsförderung ernannt werden, welche über den nötigen interdisziplinären Horizont verfügen. Diese sollten zumindest auch eine Grundausbildung in Philosophie, genauer in philosophischer Ethik haben.

4. Auf regionaler oder nationaler Ebene ist der Austausch zwischen den Beauftragten für Gesundheitsförderung sicherzustellen und ihre Ausbildung voranzutreiben.

5. In der Grundausbildung der Lehrkräfte sollte in grösseren zeitlichen Blöcken das Anliegen der Gesundheitsförderung eigens thematisiert werden, wobei der philosophischen Reflexion über die Schule in ihrer Beziehung zur ldee des "guten Lebens" Raum gegeben werden müsste.

6. In der Allgemeinen Didaktik und in allen Fachdidaktiken sind gesundheitsrelevante Themen umsetzungs- und anwendungsorientiert aufzugreifen und die Lehrpläne entsprechend zu gestalten.

7. Gesundheitsförderung sollte in allen kantonalen Lehrplänen zum durchgehenden Prinzip werden,

8. Schulbehörden und Schulleitungen haben bei der Planung und Gestaltung von Schulen und Schularealen dem Aspekt der Gesundheitsförderung Rechnung zu tragen.

Tabelle 5. Vom Ausschuss Lehrerbildung der EDK vorgeschlagene Massnahmen.

der Projektphase, dass kein Mitglied der an den sogenannten Austauschtagungen vertretenen Gruppe über die zeitlichen und finanziellen Ressourcen verfügte, um ein solches Forum sicherzustellen.

\section{Summary}

Health promotion in pre-service teacher training in Switzerland

School is an extraordinary setting through which to practice health promotion. Obviously the preparation of future teachers to this task is of great importance. In Switzerland, there are some models of good practice and many enthusiastic fighters for health in the field of preservice teacher training. Yet the results of a study at the swiss teacher training institutes show that about $30 \%$ of these institutions do not include health-issues in their curriculum. Furthermore, in those institutes including health-issues, the structural position of the person(s) encharged with this task is often weak and marginalized. The practical impact of the study is only partially satisfying: while the addressed authorities (Swiss Conference of Cantonal Ministers of Education, Cantonal school authorities) have translated and published the results of the study and give them some weight by formulating their own recommendations, the constitution of a network of the persons encharged with health-issues in the teacher training institutions has failed. 


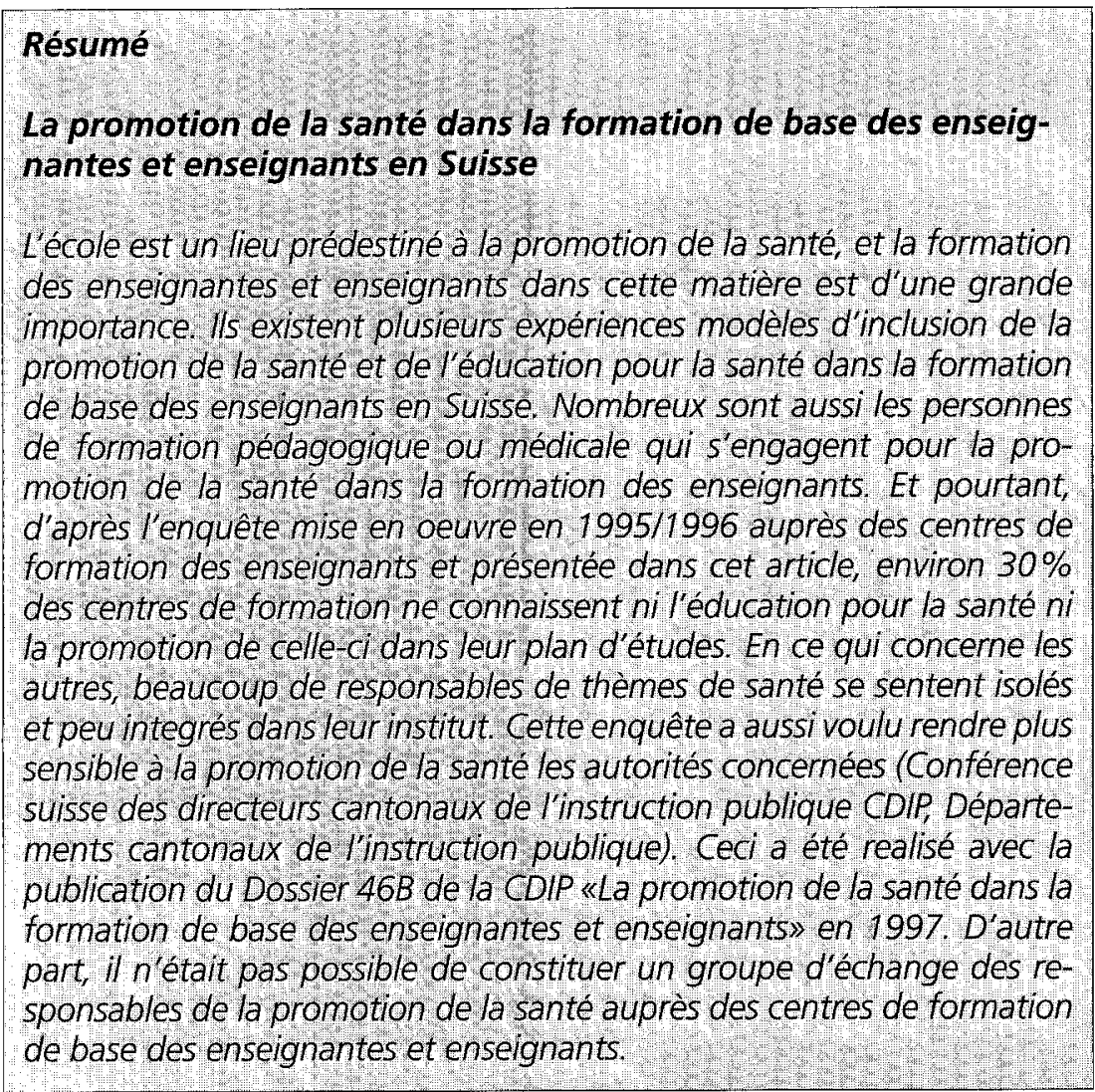

\section{Literaturverzeichnis}

1 WHO's School Health Initiative. WHO, 1995 (Arbeitspapier).

2 Gretler $S$. Gesundheitsförderung in der Grundausbildung von Lehrerinnen und Lehrern. In: Schweizerische Konferenz der kantonalen Erziehungsdirektoren, EDK-Dossier 46A, 1997.

3 Troschke J. Von der Gesundheitserziehung zur Gesundheitsförderung in der Schule. In: Priebe B, Israel G, Hurrelmann K, ed. Gesunde Schule - Gesundheitserziehung, Gesundheitsförderung, Schulentwicklung. Weinheim, Basel: Beltz, 1993: 11-38.

4 Heindl I. Gesundheitserziehung und Gesundheitsförderung in der Lehrerausbildung. In: Priebe $\mathrm{B}$, Israel G, Hurrelmann K, ed. Gesunde Schule - Gesundheitserziehung, Gesundheitsförderung, Schulentwick- lung. Weinheim Basel: Beltz, 1993: 197-208.

5 Barkholz U, Homfeldt HG. Gesundheitsförderung - Schule von innen entwickeln. In: WHO, ed. Gesundheitsförderung in Schulen. Magdeburg: WHO, 1992.

6 Campiche V, LeGauffey $Y$. Education à la santé: le point de vue des enseignants. Schweizerische Fachstelle für Alkohol- und andere Drogenprobleme, 1995.

\section{Korrespondenzadresse}

lic. phil. Simone Gretler, MPH

Institut für Ethnologie

Universität Bern

Länggassstr. $49 \mathrm{a}$

CH-3000 Bern 9

Fax +41316314212

Simone.Gretler@ethno.unibe.ch 